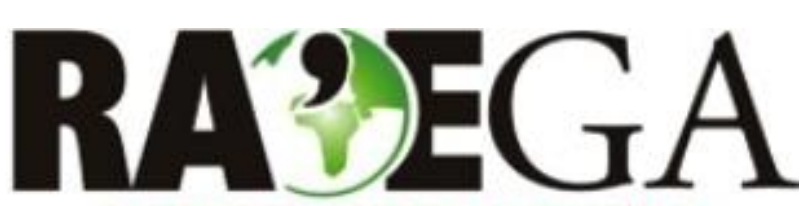

O ESPACYO GEOGRÁFICO EM ANÁLISE

\title{
O USO E A COBERTURA DA TERRA E A SUA RELAÇÃO COM A HANTAVIROSE NA REGIÃO INTEGRADA DE DESENVOLVIMENTO DO DISTRITO FEDERAL E ENTORNO
}

\section{THE USE AND LAND COVER AND ITS RELATIONSHIP WITH HANTAVIRUSES IN THE INTEGRATED DEVELOPMENT REGION OF DISTRITO FEDERAL AND SURROUNDING}

\author{
Janduhy Pereira dos Santos \\ Departamento de Geografia \\ Universidade de Brasília - UnB \\ Brasília, DF, Brasil \\ e-mail: janduhy.santos@gmail.com \\ Stefan Vilges de Oliveira \\ Secretaria de Vigilância em Saúde \\ Ministério da Saúde \\ Brasília, DF, Brasil \\ e-mail: stefan.oliveira@saude.gov.br \\ Valdir Adilson Steinke \\ Departamento de Geografia \\ Universidade de Brasília - UnB \\ Brasília, DF, Brasil \\ e-mail:valdirsteinke@gmail.com
}

\section{Recebido em: 08/06/2015}

Aceito em: 14/03/2016

\section{Resumo}

A hantavirose possui uma epidemiologia de muitas nuances com mudanças ambientais de origem antrópica. Assim, é possível perceber a necessidade de compreensão, a nível regional e local, das possíveis contribuições que as mudanças antrópicas trouxeram em relação àquela enfermidade para o Distrito Federal e seu entorno. Neste sentido, o presente estudo analisou as categorias de uso e cobertura da terra existentes na Região Integrada de Desenvolvimento (RIDE-DF) do Distrito Federal e Entorno, tendo em vista a avaliação estatística da influência destas categorias na dinâmica da hantavirose. Através do uso do Sistema de Informações Geográficas (SIG) e de testes estatísticos, como, por exemplo, o odds ratio e o qui-quadrado, foi possível avaliar a distribuição espaço-temporal da hantavirose. Dados 


\section{O USO E A COBERTURA DA TERRA E A SUA RELAÇÃO COM A HANTAVIROSE NA REGIÃO INTEGRADA DE DESENVOLVIMENTO DO DISTRITO FEDERAL E ENTORNO}

secundários de casos da doença referentes ao período 2004-2010 permitiram identificar 14 locais de ocorrência do agravo no entorno e 49 locais no Distrito Federal. Observou-se ainda nas análises que no Distrito Federal, as classes de uso - as áreas urbanas $(\mathrm{OR}=2.9)$ e as lavouras temporárias $(\mathrm{OR}=2.3)$ - que apresentaram medidas de associação significativas. Os resultados do teste Qui-quadrado apontou que os efeitos antrópicos são estatisticamente mais significativos no Distrito Federal $\left(x^{2}=9,5\right)$ do que em sua região de entorno $\left(x^{2}\right.$ $=1,8)$. Assim, 0 antropismo tem um peso importante na dispersão da hantavirose na RIDE-DF.

Palavras-chave: Hantavírus; Uso da terra; Análise espacial; Doença Transmitida por Roedores.

\section{Abstract}

The hantaviruses has an epidemiological many nuances with environmental changes of anthropogenic origin. So, you can see the need for understanding, regional and local level, the possible contributions that anthropogenic changes brought in relation to that disease for the Federal District and its surroundings. In this sense, the present study analyzed the categories of use and land cover in the Region Integrated Development (RIDE-DF) of the Federal District and surrounding areas, with a view to statistical evaluation of the influence of these categories in the dynamics of hantaviruses. Through the use of Geographic Information System (GIS) and statistical tests, for example, the odds ratio and the chi-square, it was possible to evaluate the spatial and temporal distribution of hantaviruses. Secondary data of cases of disease for the period 2004-2010 have identified 14 of the grievance occurrence sites in the surrounding area and 49 locations in the Federal District. It was also noted that the analysis in the Federal District, using classes - urban areas $(\mathrm{OR}=2.9)$ and temporary crops $(\mathrm{OR}=2.3)$ - that showed significant association measures. The results of the chi-square test indicated that the anthropogenic effects are statistically more significant in the Federal District $(x 2=9.5)$ than in its surrounding region $(x 2=$ 1.8). So the anthropism is an important factor in the spread of Hantavirus in RIDE-DF.

Keywords: Hantavirus; Land use; Spatial analysis; Rodent-Borne.

\section{INTRODUÇÃO}

Os impactos dos eventos de origem natural e humana promovem alterações dos padrões de transmissão de algumas doenças infecciosas. Tanto os fatores naturais (como, por exemplo, a temperatura, a umidade, a pluviosidade e a vegetação) e os fatores antropogênicos (as diversas formas de uso da terra) influenciam direta ou indiretamente os surtos e a disseminação destas doenças (Wu et al.,2014). 


\section{O USO E A COBERTURA DA TERRA E A SUA RELAÇÃO COM A HANTAVIROSE NA REGIÃO INTEGRADA DE DESENVOLVIMENTO DO DISTRITO FEDERAL E ENTORNO}

Como exemplo dos fatores de origem natural, observam-se os eventos climáticos que produziram resultados drásticos para a saúde pública, como, por exemplo, o episódio analisado por Acuna-Soto et al. (2002), que realizaram estudos epidemiológicos de uma febre hemorrágica conhecida como Cocoliztli, que atingiu a população nativa do México em 1545. Segundo aqueles autores, esta doença foi associada a uma forte estiagem que atingiu drasticamente a região proporcionada pelo aumento de roedores, reservatórios de um agente patogênico, causando a morte de milhões de pessoas durante o século XVI.

Outro exemplo foi a passagem do furacão Mitch na Guatemala e em El Salvador, em 1998, que provocou a morte de 9.600 pessoas, além de um surto de malária, em virtude da formação criadouros para o vetor deste agravo (FOLEY et al., 2005, p.572 apud Editorial Staff, 1999). Ademais, Glass et al. (2007) e Patz et al. (2008) reforçaram também a questão da influência de eventos climáticos na disseminação de doenças, como, por exemplo, o fenômeno do El Ninõ de 1992, que desencadeou um surto de hantavirose no sudoeste dos Estados Unidos da América (EUA). Em decorrência do excesso das chuvas esse evento propiciou uma maior produtividade de biomassa vegetal (somente alimento de roedores) e consequentemente maior densidade de reservatórios de hantavírus nessa região.

Ainda Patz et al. (2008) demonstraram que os surtos de cólera em Bangladesh estavam associados também ao fenômeno climático ENSO (Oscilação do Sul), que propiciava o aquecimento das águas do mar e que, por sua vez, favorecia o aumento da floração de algas que permitem alojar a bactéria Vibrio sp., contaminando, assim, os crustáceos e os moluscos (frutos do mar) que eram consumidos pela população, desencadeando surtos de cólera e também outras doenças entéricas.

Um dos efeitos da intervenção humana na natureza é a alteração na diversidade das comunidades de plantas, animais e microrganismos. Esta alteração pode ser representada por alguns fatores, quais sejam: redução da biodiversidade, introdução de espécies exóticas, poluição ambiental, surgimento de doenças contagiosas transmitidas por vetores e reservatórios e outros aspectos. Tal intervenção se dá principalmente pela implantação de 


\section{O USO E A COBERTURA DA TERRA E A SUA RELAÇÃO COM A HANTAVIROSE NA REGIÃO INTEGRADA DE DESENVOLVIMENTO DO DISTRITO FEDERAL E ENTORNO}

pastagens, agricultura e reflorestamentos monoespecíficos, mas também pela eliminação de áreas naturais para dar lugar às zonas urbanas, hidrelétricas, estradas e atividades de mineração.

Neste sentido, Brearley et al. (2013) descreveram que o uso da terra já foi alterado em mais de $75 \%$ da superfície terrestre, acarretando no declínio da biodiversidade em todo o mundo. Estas alterações, conforme os autores, provocaram impactos na saúde pública, como a malária, a dengue, doença de Chagas e outros. Também Gottdenker et al. (2014) descrevem que as mudanças ambientais de origem antrópica causam impactos negativos nas estruturas dos ecossistemas por perturbar os ciclos biogeoquímicos terrestres e aquáticos e, assim, alterando as propriedades daqueles ecossistemas; e ainda, introduzindo espécies não nativas e agentes patogênicos à saúde humana e animal.

Vazquez-Prokopec et al. (2006) detalham que o desequilíbrio ambiental gerado pelo desmatamento favoreceu o contato da população de triatomíneos e, consequentemente, a disseminação da doença de Chagas na região do Chaco, Argentina. Na mesma tendência, observa-se o trabalho de EstradaPenã et al. (2014), onde a exposição ao carrapato Ixodes scapularis reservatório da bactéria Borrelia burgdorferi, causadora da doença de Lyme apresentou aumento dos casos da doença em razão da fragmentação das áreas florestais na América do Norte.

A hantavirose apresenta uma epidemiologia que possui correlação com as mudanças ambientais de origem antrópica. Neste sentido, Lambin et al. (2010) abordaram a Epidemiologia Paisagística da hantavirose na Bélgica destacando a seguinte questão: em ambientes alterados por práticas agrícolas têm-se maior abundância de roedores reservatórios contaminados do que em áreas silvestres. Já Goodin et al. (2006) realizaram a correlação entre o uso e a cobertura da terra e os casos de hantavirose no Paraguai, onde perceberam que as áreas destinadas à agricultura são as mais propensas ao desenvolvimento da patologia.

No Brasil, têm-se os trabalhos pioneiros de Santos et al. (2011), que analisaram os impactos do uso da terra e a hantavirose no Distrito Federal, e 


\section{O USO E A COBERTURA DA TERRA E A SUA RELAÇÃO COM A HANTAVIROSE NA REGIÃO INTEGRADA DE DESENVOLVIMENTO DO DISTRITO FEDERAL E ENTORNO}

de Brito (2012), que fez um estudo da distribuição espacial da hantavirose no Estado de Minas Gerais e sua relação com o uso da terra naquela região. Ambos os trabalhos mencionaram que os desmatamentos ocasionados pela agricultura, pecuária e a suburbanização têm influenciado a dispersão de roedores, acarretando no surgimento de surtos esporádicos de hantavirose naquelas localidades.

Santos et al. (2012) abordaram os impactos da rodovia BR-163 (CuiabáSantarém) sobre o meio ambiente ao longo da rodovia e perceberam que as alterações espaciais relatadas colaboraram também no aumento dos casos de hantavirose. Neste sentido, têm-se aí um esforço na compreensão dos processos espaciais que interferem no ciclo das doenças infecciosas transmitidas por animais vetores e reservatórios de patógenos dos hantavírus presentes no território nacional.

Santos (2008) abordou o fato de que os espaços naturais vêm sendo substituídos por ambientes artificiais que trazem consequências à saúde pública, uma vez que tais ambientes tornaram-se ambientes hostis. Trazendo para a questão da hantavirose, é possível observar que para aquele autor, o espaço habitado alcançou uma situação-limite, onde o processo destrutivo pode se tornar irreversível. Neste sentido, na medida em que o uso do solo se torna intensivo em razão das atividades agropecuárias e da suburbanização, maiores serão os efeitos sobre o ambiente e, por sua vez, sobre a biodiversidade.

A partir das referidas considerações é possível notar a necessidade de compreender, a nível regional e local, as possíveis contribuições que as mudanças antrópicas trouxeram em relação à hantavirose para o Distrito Federal e sua região de entorno, formada por alguns Municípios dos Estados de Goiás e Minas Gerais, pois, conforme trabalhos descritos por Santos et al. (2011) e Oliveira et al. (2013), aquelas regiões apresentam o bioma do cerrado, além de quantidade considerável de casos hantavirose, fazendo com que o Ministério da Saúde (2005) considere de suma importância os estudos dos fatores ecoepidemiológicos e socioeconômicos, como, por exemplo, a 


\section{O USO E A COBERTURA DA TERRA E A SUA RELAÇÃO COM A HANTAVIROSE NA REGIÃO INTEGRADA DE DESENVOLVIMENTO DO DISTRITO FEDERAL E ENTORNO}

ocupação humana recente de áreas de cerrado ou o aumento da população de roedores reservatórios nestas áreas.

Diante do exposto, o presente estudo teve como objetivo analisar as categorias de uso e cobertura da terra presentes na Região Integrada de Desenvolvimento do Distrito Federal e Entorno (RIDE-DF), período 2004-2010, tendo em vista avaliar espacialmente a influência destas categorias na dinâmica da hantavirose e, assim, subsidiar as ações de saúde pública para o controle do agravo.

\section{MATERIAIS E MÉTODOS}

\section{1 Área de estudo}

A área analisada encontra-se entre os meridianos $47^{\circ} 00^{\prime}$ e $49^{\circ} 00^{\prime}$ de longitude oeste e os paralelos $15^{\circ} 00^{\prime}$ e $17^{\circ} 00^{\prime}$ de latitude sul, inserida na região conhecida como RIDE-DF, e abrange uma área de 55.402,2 km². É constituída (Figura 1) pelo Distrito Federal, pelos Municípios de Abadiânia, Água Fria de Goiás, Águas Lindas de Goiás, Alexânia, Cabeceiras, Cidade Ocidental, Cocalzinho de Goiás, Corumbá de Goiás, Cristalina, Formosa, Luziânia, Mimoso de Goiás, Novo Gama, Padre Bernardo, Pirenópolis, Planaltina, Santo Antônio do Descoberto, Valparaíso de Goiás e Vila Boa, no Estado de Goiás, e pelas localidades de Unaí, Buritis e Cabeceira Grande, no Estado de Minas Gerais.

Segundo as informações provenientes do Ministério da Integração Nacional (MI) (2014), a RIDE-DF foi criada em 1998 pela Lei Complementar oㅜ 94, de 19 de fevereiro de 1998, e regulamentada pelo Decreto $n^{\circ} 7.469$, de 04 de maio de 2011, com o objetivo de promover a articulação da ação administrativa da União, dos Estados de Goiás e de Minas Gerais e do Distrito Federal, a fim de reduzir as desigualdades regionais causadas pela alta concentração urbana decorrente do fluxo migratório entre o Distrito Federal e os municípios vizinhos, bem como a pressão existente em relação aos serviços públicos oferecidos pela capital do país. 


\section{O USO E A COBERTURA DA TERRA E A SUA RELAÇÃO COM A HANTAVIROSE NA REGIÃO INTEGRADA DE DESENVOLVIMENTO DO DISTRITO FEDERAL E ENTORNO}

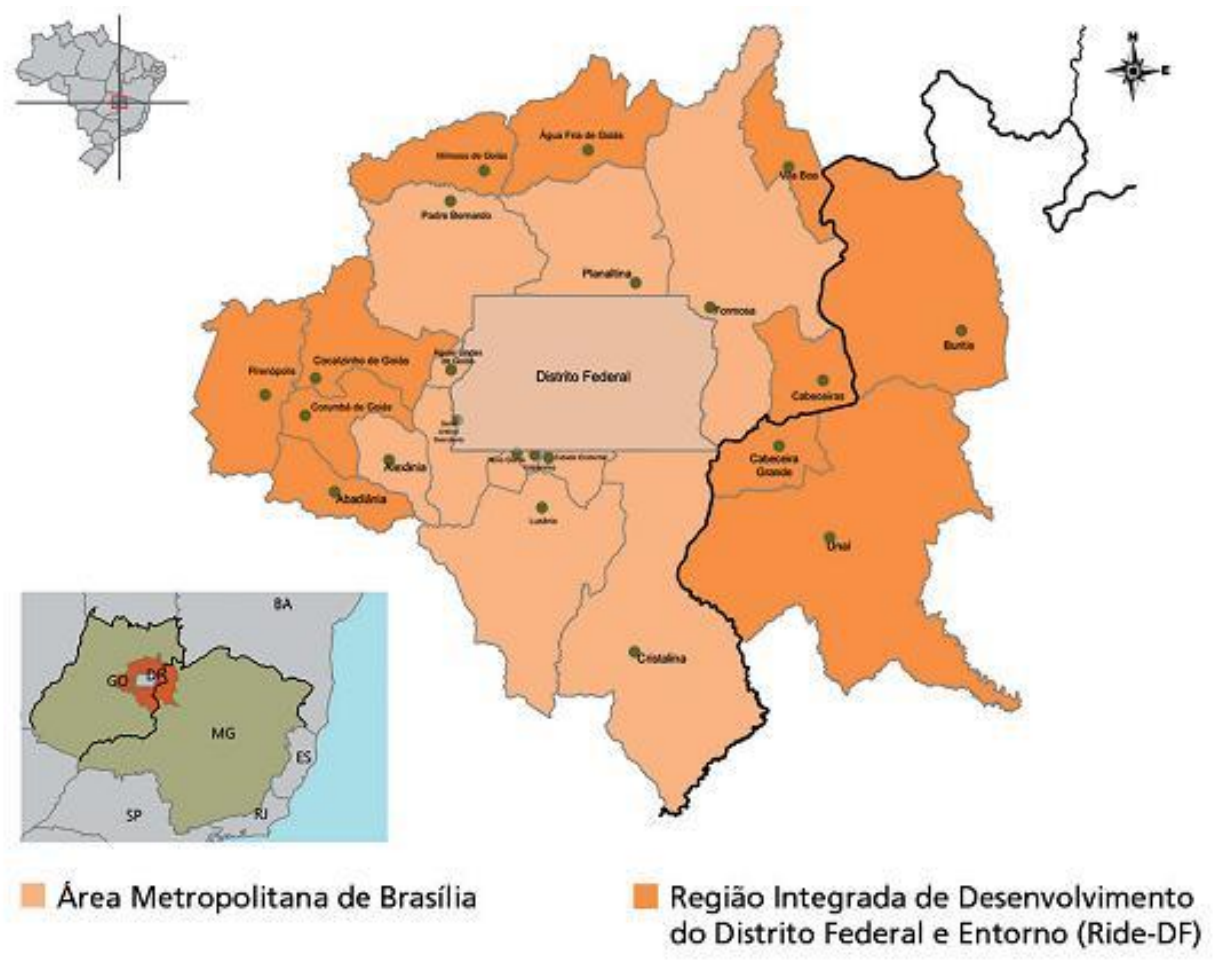

FIGURA 1 - Mapa de localização da Região Integrada de Desenvolvimento do Distrito Federal e Entorno (RIDE-DF).

Fonte: Instituto Brasileiro de Geografia e Estatística (IBGE). Malha municipal 2010. Elaboração: Companhia de Planejamento do Distrito Federal (CODEPLAN).

Para o Instituto de Pesquisas Econômicas Aplicadas (IPEA) (2014), em 2010, a RIDE-DF possuía um grau de urbanização de $94,1 \%$. A população do município núcleo (Distrito Federal) desta correspondia, em 2010, a 69\% da população da região. A taxa de crescimento da população da RIDE-DF, entre 2000 e 2010 , foi de $2,33 \%$ ao ano.

\subsection{Dados epidemiológicos}

A população do presente estudo foi constituída pela totalidade dos casos confirmados de hantavirose notificados ao MS dos Municípios integrantes (Entorno) da RIDE-DF e das Regiões Administrativas (RAs) do Distrito Federal no período 2004-2010 no Sistema de Informação de Agravos de Notificação (SINAN). As informações foram produzidas por um instrumento de coleta de dados, denominado de Ficha de Investigação Epidemiológica (FIE) de hantavirose. Os dados do SINAN são gerados pelas Secretarias Municipais de Saúde (SMS), seguindo para as Secretarias Estaduais de Saúde (SES) e, posteriormente, enviadas para o âmbito nacional, para a área técnica das 


\section{O USO E A COBERTURA DA TERRA E A SUA RELAÇÃO COM A HANTAVIROSE NA REGIÃO INTEGRADA DE DESENVOLVIMENTO DO DISTRITO FEDERAL E ENTORNO}

hantaviroses, por meio eletrônico. Estes dados foram analisados em relação às possíveis duplicidades e incoerências, sendo que as tabulações foram efetuadas através do software Microsoft Office Excel $2007^{\circledR}$.

As fichas que tiveram todos os campos preenchidos foram aproveitadas com o intuito de determinar as áreas de ocorrências dos casos confirmados. Os dados reunidos foram organizados em planilhas do programa OpenOffice.org $\mathrm{Calc}^{\circledast}$ para georreferenciamento dos locais de ocorrência com as coordenadas latitude/longitude em graus decimais com o Datum World Geodetic System 84. A conversão no formato shapefile se deu pelo software ArcGIS, versão 10.2.2, desenvolvido pela Environmental System Research Institute ${ }^{\circledR}$.

\subsection{Base cartográfica do uso e cobertura da Terra da Região Integrada de Desenvolvimento do Distrito Federal e Entorno.}

A base cartográfica digital do uso e da cobertura da terra foi obtida de 02 (dois) bancos de dados governamentais. Um dos bancos de dados utilizados foi o do Instituto Brasileiro de Geografia e Estatística (IBGE), que disponibilizou a malha digital (formato shapefile) dos Estados de Goiás, Minas Gerais e do Distrito Federal para composição do mapa da RIDE-DF, com sistema de coordenadas geográficas (latitude e longitude), escala de 1:1.000.000 e Datum em SIRGAS 2000 e posteriormente convertido para o Datum World Geodetic System 84.

O outro banco de dados governamental foi obtido junto ao Ministério do Meio Ambiente (MMA), que realizou através do Departamento de Zoneamento Territorial a classificação de uso e da ocupação do solo através do diagnóstico físico-biótico do Zoneamento Ecológico e Econômico da RIDE-DF. O tema foi atualizado em 2007, a partir de imagens orbitais provenientes do sensor HRC (Câmera Pancromática de Alta Resolução) do satélite CBERS-2B do Instituto Nacional de Pesquisas Espaciais (INPE). As imagens no formato raster foram convertidas posteriormente no formato shapefile.

A malha digital possui um sistema de coordenadas geográficas (latitude e longitude), escala de 1:250.000 e Datum em South America 69 que posteriormente foi padronizado para o World Geodetic System 84. 


\section{O USO E A COBERTURA DA TERRA E A SUA RELAÇÃO COM A HANTAVIROSE NA REGIÃO INTEGRADA DE DESENVOLVIMENTO DO DISTRITO FEDERAL E ENTORNO}

Com o intuito de facilitar a compreensão daquele mapa, fez-se uma divisão em 02 (duas) categorias, quais sejam: 1) classes de uso; e, 2) cobertura da terra. A categoria antrópica apresenta as classes de uso da terra, ou seja, são aquelas que possuem suas estruturas espaciais modificadas pela ação humana, e a categoria não antrópicos refere-se às classes de cobertura da terra, isto é, as estrutura espaciais formadas por elementos naturais.

Através da classificação supervisionada, foi possível a discriminação das seguintes classes: antrópicas (aeroporto, área urbana, estação de tratamento de esgoto, lavoura temporária, lavoura permanente, pastagem e vegetação secundária, pivô e reflorestamento) e não antrópicas (floresta galeria, lago, savana florestada, savana parque, savana parque/floresta sazonal montana, savana parque/gramínea-lenhosa, savana parque/savana arborizada, savana parque/savana floresta e solo exposto).

Para o tratamento, a organização e a interação dos dados numéricos e gráficos em ambiente de Sistemas de Informações Geográficas (SIG) foi utilizado o software ArcGIS, versão 10.2.2 ${ }^{\circledR}$, desenvolvido pela Environmental System Research Institute (ESRI).

\subsection{Análise de Odds Ratio}

Para a análise estatística da associação das variáveis de cobertura da terra com os casos de hantavirose na área de estudo optou-se pela análise de Odds Ratio ou Razão de Chances, cujo teste avalia as proporções dispostas em tabela de contingência $2 \times 2$, ou seja, para 02 (duas) amostras dicotomizadas, mensuradas a nível nominal, onde se tem o cálculo da vantagem (ou desvantagem) de um dos eventos - sucesso - em relação ao outro (AYRES et al., 2007).

Se as probabilidades de um evento em cada um dos grupos forem $p$ (primeiro grupo) e $q$ (segundo grupo), então, a razão de chances se dá como se segue:

$$
\frac{p /(1-p)}{q /(1-q)}=\frac{p(1-q)}{q(1-p)}
$$

Uma razão de chances de 1 indica que a condição ou evento sob estudo é igualmente provável de ocorrência nos dois grupos. Uma razão de chances 


\section{O USO E A COBERTURA DA TERRA E A SUA RELAÇÃO COM A HANTAVIROSE NA REGIÃO INTEGRADA DE DESENVOLVIMENTO DO DISTRITO FEDERAL E ENTORNO}

maior do que 1 indica que a condição ou evento tem maior chance de ocorrer no primeiro grupo. Finalmente, uma razão de chances menor do que 1 indica que a probabilidade é menor no primeiro grupo do que no segundo grupo.

Análise de Odds Ratio no estudo de caso e controle entre a variável de uso e cobertura da terra com casos de hantavirose e a quantidade de áreas dessa variável e a variável de uso e cobertura da terra, com casos de hantavirose e sem a quantidade de áreas, no mesmo período. Para a realização desta análise, o software utilizado foi o BioEstat, versão $5.0^{\circledR}$, desenvolvido pela Universidade Federal do Pará (UFPA).

\subsection{Análise pelo teste de Qui-quadrado $\left(x^{2}\right)$}

É um teste não paramétrico, ou seja, os parâmetros média e desviopadrão não são utilizados. Neste, as frequências de cada grupo são relacionadas em uma tabela de dupla entrada. O teste do Qui-Quadrado deve ser aplicado quando cada frequência for um número maior ou igual a 5 . Se a frequência de alguma das células da tabela for menor que 5 , esta deverá ser agrupada com outra classe (AYRES et al., 2007).

Esse teste deve ser aplicado quando as variáveis tiverem no mínimo 02 (duas) categorias. E a hipótese deste tipo de teste é dada por:

$\left\{\begin{array}{l}\mathrm{H}_{0}: \mathrm{y} \text { independe de } \mathrm{x} \\ \mathrm{H}_{1}: \mathrm{y} \text { não independe de } \mathrm{x}\end{array}\right.$

$$
X^{2}=\sum_{i=j}^{k=1} \frac{\left(f_{o}-f_{e}\right)^{2}}{f_{e}}
$$

$g \mathrm{l}=c-1 \times l-1$

Onde: $f_{0}=$ frequência observada; $f_{\mathrm{e}}=$ frequência esperada.

Para a realização das análises citadas, fez-se uso do software BioEstat, versão $5.0^{\circledR}$, desenvolvido pela UFPA.

\section{RESULTADOS}

Os dados obtidos analisaram 179 casos confirmados ocorridos no Distrito Federal e Entorno do Estado do Goiás, sendo possível determinar 66 áreas de ocorrência da infecção. A Figura 2, a seguir, apresenta a 


\section{O USO E A COBERTURA DA TERRA E A SUA RELAÇÃO COM A HANTAVIROSE NA REGIÃO INTEGRADA DE DESENVOLVIMENTO DO DISTRITO FEDERAL E ENTORNO}

sobreposição dos casos de hantavirose no mapa de uso e cobertura da terra da região em estudo. Na região de entorno do Distrito Federal foram contabilizados 26 casos de hantavirose no período 2004-2010. Foi possível determinar 14 locais de ocorrência do agravo e, assim, observar uma concentração dos casos no entorno sul, principalmente nos municípios goianos de Cidade Ocidental, Cristalina, Luziânia e Valparaíso de Goiás.

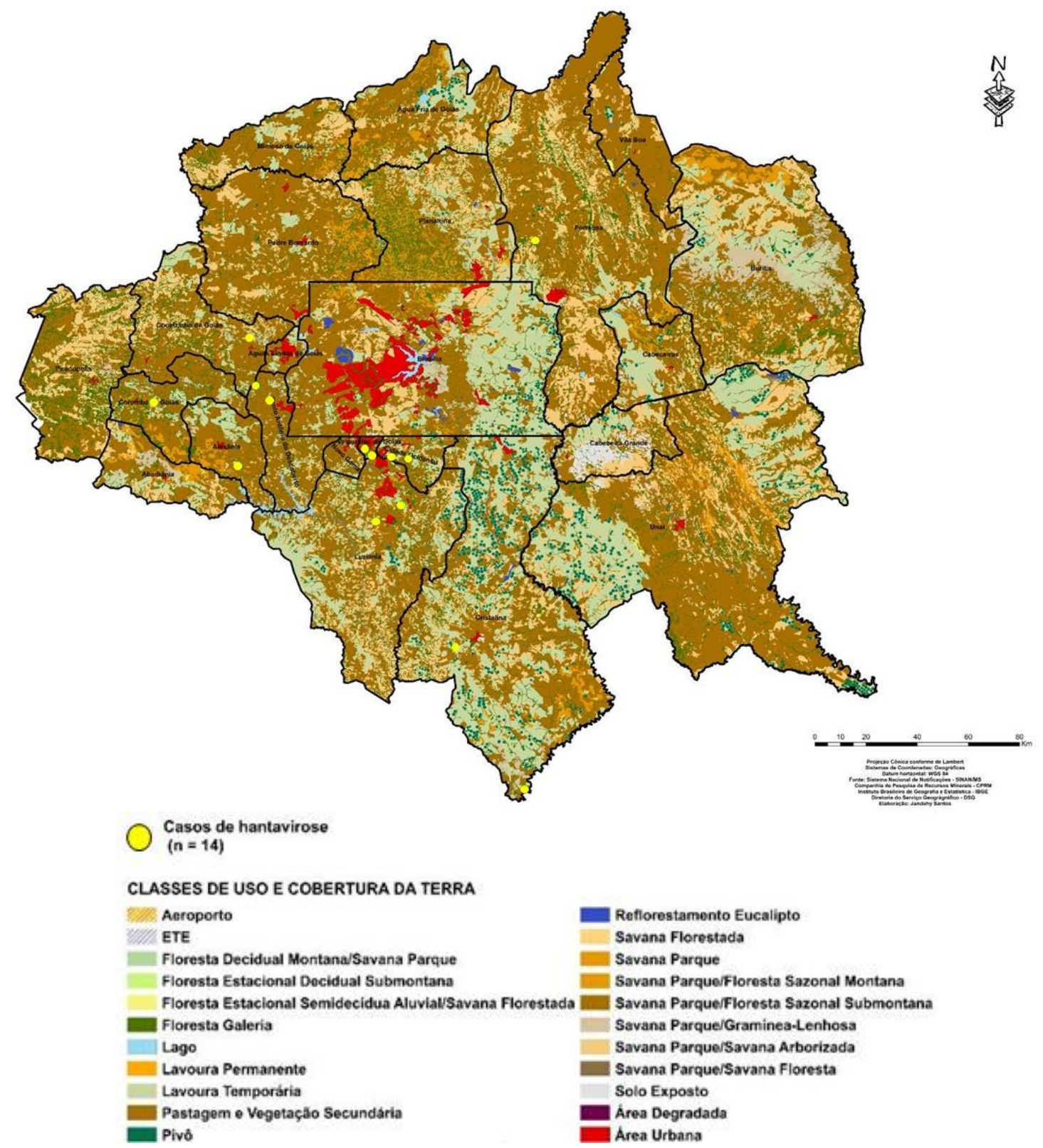

FIGURA 2 - Mapa de uso e cobertura da terra da Região Integrada de Desenvolvimento do Distrito Federal e Entorno (RIDE-DF) e os casos de hantavirose, período 2004-2010. Elaboração: Janduhy Santos, 2014. 


\section{O USO E A COBERTURA DA TERRA E A SUA RELAÇÃO COM A HANTAVIROSE NA REGIÃO INTEGRADA DE DESENVOLVIMENTO DO DISTRITO FEDERAL E ENTORNO}

O cartograma apresentado anteriormente (Figura 3) aponta a diversidade de uso da terra de acordo com o levantamento realizado pelo MMA (2007). Com o intuito de facilitar a compreensão daquele mapa, fez-se uma divisão em 02 (duas) categorias, quais sejam: 1) classes de uso; e, 2) cobertura da terra. A categoria antrópica apresenta as classes de uso da terra, ou seja, são aquelas que possuem suas estruturas espaciais modificadas pela ação humana, e a categoria não antrópicos refere-se às classes de cobertura da terra, isto é, as estrutura espaciais formadas por elementos naturais.

Neste sentido, conforme a organização dos dados nos SIG e o mapa apresentado na Figura 3 é possível observar as agregações (classes) de uso e cobertura da terra existentes na região e a quantidade de casos por classe na Tabela 1, que se segue:

Tabela 1 - Área total das classes de uso e cobertura da terra do Entorno e os casos de hantavirose, período 2004-2010.

\begin{tabular}{|c|c|c|}
\hline Classe & $\begin{array}{l}\text { Área coberta } \\
\left(\mathrm{Km}^{2}\right)\end{array}$ & $\begin{array}{c}\text { Casos de } \\
\text { hantavirose }\end{array}$ \\
\hline \multicolumn{3}{|c|}{ Antrópicos (Uso da terra) } \\
\hline Área degradada & - & - \\
\hline Área urbana & 327,61 & - \\
\hline Lavoura permanente & 15,83 & - \\
\hline Lavoura temporária & 9.830 .73 & 3 \\
\hline Pastagem e vegetação secundária & $24.840,68$ & 6 \\
\hline Reflorestamento & 100,52 & - \\
\hline \multicolumn{3}{|c|}{ Não antrópicos (Cobertura da terra) } \\
\hline Floresta decidual montana/savana parque & 42,12 & - \\
\hline Floresta estacional decídua/submontana & 35,09 & - \\
\hline Floresta estacional semidecídua aluvial/savana florestada & 41,14 & - \\
\hline Floresta galeria & $5.939,99$ & 2 \\
\hline Lago & 377,32 & - \\
\hline Savana florestada & 149,46 & - \\
\hline Savana parque & 875,52 & 1 \\
\hline Savana parque/floresta sazonal montana & $1.010,62$ & - \\
\hline Savana parque/floresta sazonal submontana & 825,41 & - \\
\hline Savana parque/gramínea-lenhosa & $1.021,43$ & - \\
\hline Savana parque/savana arborizada & $8.706,84$ & 1 \\
\hline
\end{tabular}


Conforme a tabela 1 apresentada anteriormente, os espaços antropogênicos, como, por exemplo, as pastagens, apresentaram 49\% dos casos de hantavirose na região analisada no período 2004-2010. Tais espaços são compostos por áreas destinadas ao pastoreio do gado, formados mediante plantio de forragens perenes ou aproveitamento e melhoria de pastagens naturais. A Figura 2 também apresentou, em associação às pastagens, a vegetação secundária - formação resultante de processos naturais de sucessão, após supressão total ou parcial de vegetação primária por ações antrópicas ou causas naturais.

As áreas antrópicas agrícolas representadas pela lavoura temporária responderam por $21 \%$ dos casos de hantavirose. A referida classe é formada por cultivo de plantas de curta ou média duração, em geral, com ciclo vegetativo inferior a um ano, que, após a produção, deixam o terreno disponível para novo plantio. Já a classe lavoura permanente é compreendida pelo cultivo de plantas perenes, isto é, de ciclo vegetativo de longa duração. Estas plantas produzem por vários anos sucessivos sem a necessidade de novos plantios após colheita. Neste sentido, esta última classe não apresentou casos de hantavirose plotados em suas áreas no mapa (Figura 2).

$E$ ainda, os reflorestamentos, que na Tabela 1 são representados pelo plantio de espécies exóticas, tais como: eucalipto, pínus e acácia-negra. Em ambas as classes não foram observadas ocorrências de hantavírus. E o mesmo se dá para as áreas degradadas que sofreram processos de deterioração provocada pelas atividades humanas, como, por exemplo, a extração de minerais.

As coberturas florísticas existentes nos municípios integrantes do Entorno são caracterizadas por formações savânicas em razão dos solos areníticos lixiviados e ricos em alumínio. Já as formações florestais estacionais que se expandiram através da rede hidrográfica encontram-se em áreas como solos ígneos e logicamente mais férteis (IBGE, 2013). Em relação às coberturas que apresentaram ocorrências de hantavirose foi possível observar 


\section{O USO E A COBERTURA DA TERRA E A SUA RELAÇÃO COM A HANTAVIROSE NA REGIÃO INTEGRADA DE DESENVOLVIMENTO DO DISTRITO FEDERAL E ENTORNO}

que a cobertura representada pela floresta de galeria apresentou $14 \%$ das ocorrências.

Com 7\% das ocorrências de hantavirose, as formações campestres do tipo savana parque (campo sujo), savana parque/savana arborizada (cerrado stricto sensu) e savana parque/savana florestada (cerradão) foram as áreas que apresentaram tais ocorrências. Em razão da escala utilizada (1:250.000), a Figura 2 apresentou a conjugação da savana parque com a savana arborizada, bem como a savana floresta ou florestada.

Os solos expostos podem ser de origem natural, como é o caso das áreas cobertas por rocha nua exposta, ou não, como, por exemplo, as áreas de extração abandonadas. Em relação àqueles espaços, não foram constatados casos de hantavirose.

Seguindo os mesmos princípios do mapa da Figura 2, o mapa da Figura 3, a seguir, detalha os processos de uso da terra e os casos sobrepostos de hantavirose no Distrito Federal na escala temporal do estudo. Dos 78 casos autóctones confirmados no Distrito Federal foram identificados 49 locais de ocorrência para a hantavirose. 


\section{O USO E A COBERTURA DA TERRA E A SUA RELAÇÃO COM A HANTAVIROSE NA REGIÃO INTEGRADA DE DESENVOLVIMENTO DO DISTRITO FEDERAL E ENTORNO}

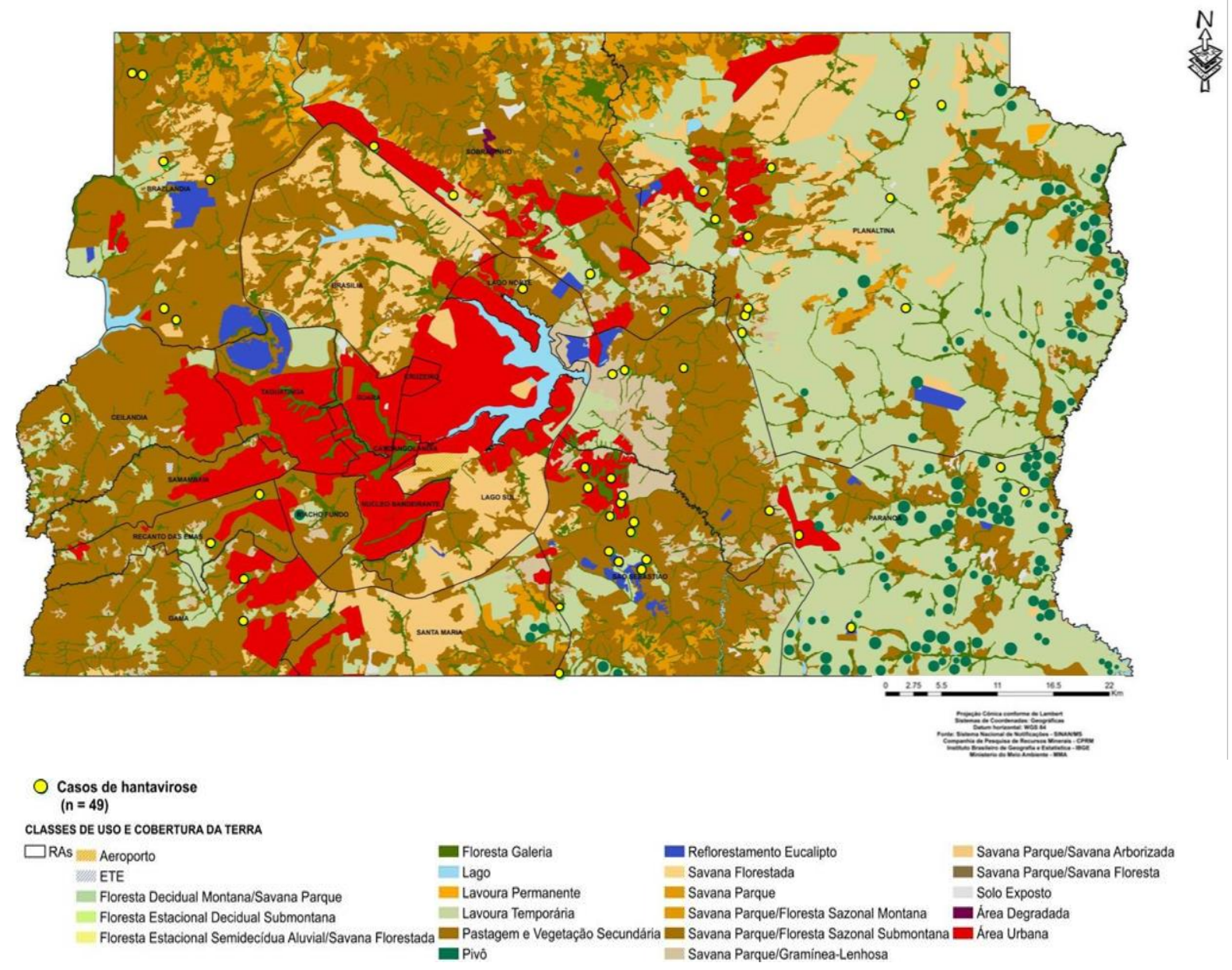

Figura 3 - Mapa de uso e cobertura da terra do Distrito Federal e os casos de hantavirose, período 2004-2010. Elaboração: Janduhy Santos, 2014.

$\mathrm{Na}$ Tabela 2, a seguir, têm-se as agregações de uso e ocupação da terra predominantes no Distrito Federal e representadas no mapa da Figura 4. 


\section{O USO E A COBERTURA DA TERRA E A SUA RELAÇÃO COM A HANTAVIROSE NA REGIÃO INTEGRADA DE DESENVOLVIMENTO DO DISTRITO FEDERAL E ENTORNO}

Tabela 2 - Área total das classes de uso e cobertura da terra no Distrito Federal e os casos de hantavirose, período 2004-2010.

\begin{tabular}{|c|c|c|}
\hline Classe & $\begin{array}{l}\text { Área coberta } \\
\qquad\left(\mathrm{Km}^{2}\right)\end{array}$ & $\begin{array}{l}\text { Casos de } \\
\text { hantavirose }\end{array}$ \\
\hline \multicolumn{3}{|c|}{ Antrópicos (Uso da terra) } \\
\hline Área degradada & 1,8 & - \\
\hline Área urbana & 647,08 & 5 \\
\hline Lavoura permanente & 5,0 & - \\
\hline Lavoura temporária & $1.605,7$ & 14 \\
\hline Pastagem e vegetação secundária & 2.089 & 20 \\
\hline Reflorestamento & 70,7 & 2 \\
\hline \multicolumn{3}{|c|}{ Não antrópicos (Cobertura da terra) } \\
\hline Floresta decidual montana/savana parque & - & - \\
\hline Floresta estacional decídua/submontana & - & - \\
\hline Floresta estacional semidecídua aluvial/savana florestada & - & - \\
\hline Floresta galeria & 389,35 & 2 \\
\hline Lago & 60,8 & - \\
\hline Savana florestada & 0,31 & - \\
\hline Savana parque & 41,6 & - \\
\hline Savana parque/floresta sazonal montana & 162,20 & - \\
\hline Savana parque/floresta sazonal submontana & - & - \\
\hline Savana parque/gramínea-lenhosa & 148,66 & 2 \\
\hline Savana parque/savana arborizada & 550,22 & 4 \\
\hline Savana parque/savana floresta & 0,21 & - \\
\hline Solo exposto & 15,3 & - \\
\hline
\end{tabular}

Para uma análise da associação das ocorrências de hantavirose com as classes de uso da terra foi calculado o Odds Ratio (OR) das categorias envolvidas com aquela enfermidade, a fim de avaliar a intensidade de associação no Distrito Federal e no Entorno, conforme exposto na Tabela 3, a seguir. 


\section{O USO E A COBERTURA DA TERRA E A SUA RELAÇÃO COM A HANTAVIROSE NA REGIÃO INTEGRADA DE DESENVOLVIMENTO DO DISTRITO FEDERAL E ENTORNO}

Tabela 3 - Resultados da análise da relação do uso da terra e a ocorrência de hantavirose no Distrito Federal e no Entorno, período 2007-2010.

\begin{tabular}{|c|c|c|c|c|}
\hline \multirow{2}{*}{ Classe de uso e cobertura da terra } & \multicolumn{2}{|c|}{ № de áreas } & \multirow{2}{*}{$P$ value } & \multirow{2}{*}{ OR $(95 \% \mathrm{Cl})$} \\
\hline & Caso & Controle & & \\
\hline \multicolumn{5}{|l|}{ ENTORNO } \\
\hline Lavoura temporária & 3 & 2.438 & 0,21 & $2,90(0,80-10,4)$ \\
\hline Pastagem e vegetação secundária & 6 & 8.139 & 0,38 & $1,8(0,64-5,37)$ \\
\hline Savana parque/savana floresta & 1 & 293 & 0,34 & $7,31(0,96-56,5)$ \\
\hline Savana parque & 1 & 498 & 0,60 & $4,30(0,56-32,9)$ \\
\hline Savana parque/savana arborizada & 1 & 7.114 & 0,21 & $0,22(0,03-1,75)$ \\
\hline Floresta galeria & 2 & 4.689 & 0,89 & $0,84(0,18-3,76)$ \\
\hline \multicolumn{5}{|l|}{ DISTRITO FEDERAL } \\
\hline Área urbana & 5 & 114 & 0,04 & $2,93(1,14-7,54)$ \\
\hline Lavoura temporária & 14 & 453 & 0,01 & $2,30(1,22-4,31)$ \\
\hline Pastagem e vegetação secundária & 20 & 1.014 & 0,20 & $1,51(0,85-2,67)$ \\
\hline Reflorestamento & 2 & 31 & 0,16 & $4,15(0,96-17,8)$ \\
\hline Floresta galeria & 2 & 387 & 0,11 & $0,29(0,07-1,21)$ \\
\hline Savana parque/gramínea-lenhosa & 2 & 211 & 0,62 & $0,57(0,13-2,38)$ \\
\hline Savana parque/savana arborizada & 4 & 323 & 0,75 & $0,75(0,26-2,10)$ \\
\hline
\end{tabular}

${ }^{*} \mathrm{OR}=$ odds ratio; $\mathrm{Cl}=$ intervalo de confiança

A análise exposta na Tabela 3 aponta que no entorno, nenhuma classe de cobertura e uso da terra apresentou resultados estatisticamente significativos para o Odds Ratio dentro do $p$-valor de $<0.05$. No Distrito Federal, as classes de uso - as áreas urbanas $(\mathrm{OR}=2.9)$ e as lavouras temporárias $(\mathrm{OR}=2.3)$ - que apresentaram medidas de associação significativas.

Com base em tais variações, a Tabela 4, a seguir, apresenta os resultados do teste do Qui-Quadrado $\left(\mathrm{X}^{2}\right)$, a fim de avaliar se a hantavirose do Distrito Federal e da RIDE-DF possui alguma dependência em relação a hantavirose com $p$-valor de $<0.05$.

Tabela 4 - Resultados do teste do Qui-Quadrado $\left(\mathrm{X}^{2}\right)$ para o Distrito Federal e no Entorno, período 2007-2010.

\begin{tabular}{llcc}
\multicolumn{1}{c}{ Região } & $\mathrm{X}^{2}$ & $\mathrm{x}^{2}$ Yates & $p$-valor \\
\hline Distrito Federal & 9,5 & 8,5 & 0,0034 \\
Entorno & 1,8 & 1,0 & 0,30 \\
\hline
\end{tabular}




\section{O USO E A COBERTURA DA TERRA E A SUA RELAÇÃO COM A HANTAVIROSE NA REGIÃO INTEGRADA DE DESENVOLVIMENTO DO DISTRITO FEDERAL E ENTORNO}

Para a os municípios que integram o entorno, os resultados do teste Quiquadrado obtidos e devidamente corrigidos (Yates) não são estatisticamente significativos, ou seja, a análise sugere que a ocorrência de hantavirose independe dos fatores de uso da terra. Já para o Distrito Federal, conforme a tabela apresentada anteriormente, se deu o inverso em relação ao entorno: o resultado do $\mathrm{X}^{2}$ apresentou dados estatisticamente significativos, rejeitando, assim, a hipótese de nulidade e aceitando a alternativa de que a ocorrência da hantavirose no Distrito Federal possui alguma dependência com o uso da terra para o período 2004-2010.

\section{DISCUSSÃO}

Com base nos resultados, os espaços antropizados do entorno são os mais predominantes com as pastagens como a maior estrutura espacial da região. Estas paisagens antropizadas oferecem condições propícias para 0 contato do homem com o hantavírus - contato percebido através das atividades agrícolas, onde o roedor é atraído pela oferta de grãos que são consumidos principalmente na época da seca, quando se tem a diminuição de insetos e sementes em seu habitat.

Observando os valores do Odds ratio e do Qui-quadrado percebe-se que nos municípios que integram o entorno do Distrito Federal, tanto as classes de cobertura como as de uso não ofereceram valores que apresentassem significação estatística a um nível de $5 \%$ para considerá-los como fator de importância na transmissão da hantavirose. Tal fato se deve pela quantidade de ocorrências disponíveis que não permitiram fornecer informações conclusivas, sendo necessário o cruzamento com outras referências e bases de dados para a obtenção de outras complementações.

Em relação ao Distrito Federal, as áreas urbanas e as áreas destinadas às lavouras temporárias tiveram o maior Odds Ratio. As áreas urbanizadas no Distrito Federal vêm apresentando nos últimos anos uma grande expansão, o que aumenta a fragmentação dos espaços silvestres existentes no território. Tal expansão se dá com o aumento de empreendimentos imobiliários para todas as classes da população. Têm-se aí o surgimento de condomínios de 


\section{O USO E A COBERTURA DA TERRA E A SUA RELAÇÃO COM A HANTAVIROSE NA REGIÃO INTEGRADA DE DESENVOLVIMENTO DO DISTRITO FEDERAL E ENTORNO}

luxo que, muitas vezes, se instalam em áreas próximas às matas em razão dos aspectos estéticos e de elevada qualidade ambiental; e ainda, têm-se a implantação de assentamentos urbanos regularizados ou surgimento de aglomerados subnormais (favela, invasão, grota, baixada, comunidade, vila, ressaca, mocambo, palafita e outros) em áreas periféricas próximas aos ambientes silvestres. A questão da expansão habitacional, sem dúvida, é um aspecto bem característico do Distrito Federal, e isso se dá em razão da diluição que ocorre das áreas rurais e silvestres em favor da criação de novos projetos urbanos, tornando difícil delimitar o que é área urbana e o que é área rural.

Contudo, $83 \%$ dos casos de hantavirose no Distrito Federal se deram em áreas antrópicas. $E$ ainda, as áreas destinadas para as pastagens e vegetação secundária representaram $41 \%$, e as lavouras temporárias apresentaram 28\% do agravo. As áreas urbanas, por sua vez, corresponderam a 10\% de presença do agravo em seus espaços. Em relação à classe urbana, o Ministério da Saúde (2013) detalha que a expansão das cidades tem trazido consigo a construção de moradias em regiões rurais, agrícolas e silvestres que circundam os Municípios. Neste sentido, vale ressaltar que casos associados a tal fator foram detectados, por exemplo, nos Estados de Minas Gerais, Mato Grosso, São Paulo e no Distrito Federal (BRITO, 2012; SANTOS, 2011). Os levantamentos de uso e cobertura da terra fornecem subsídios para as análises e avaliações dos impactos ambientais (provenientes de desmatamentos, perda da biodiversidade, mudanças climáticas, doenças reincidentes) ou de inúmeros impactos gerados pelos altos índices de urbanização e pelas transformações rurais (IBGE, 2013).

Ademais, a perda do habitat em virtude do desmatamento para o plantio de culturas temporárias ou permanentes e de pastagens desaloja aqueles roedores de seu habitat, fazendo-os procurarem abrigo em tocas próximas às benfeitorias (paióis, galinheiros, silos e outros) dos estabelecimentos agropecuários; e ainda, sendo animais com comportamento oportunista, infiltram-se em tais ambientes para se alimentarem, eliminando, por sua vez, 


\section{O USO E A COBERTURA DA TERRA E A SUA RELAÇÃO COM A HANTAVIROSE NA REGIÃO INTEGRADA DE DESENVOLVIMENTO DO DISTRITO FEDERAL E ENTORNO}

excrementos contaminados com o hantavírus que se misturam com a poeira (SANTOS, 2011).

O resultado do teste Qui-Quadrado apresentou um valor relativamente alto, ao contrário do entorno, que apresentou uma taxa relativamente baixa em razão da quantidade de locais de ocorrência disponíveis para avaliação. Neste sentido, através do resultado do teste de Qui-Quadrado, é possível observar que o antropismo tem contribuído na dinâmica espacial da hantavirose no Distrito Federal.

\section{CONSIDERAÇÕES FINAIS}

Após as análises dos dados epidemiológicos e espaciais dos casos de hantavirose na no Distrito Federal e no seu entorno, constatou-se um arranjo espacial sui generis da doença na região.

Apesar de não terem apresentado resultados estatisticamente expressivos, as formações florísticas do bioma Cerrado: a savana parque (campo-sujo) e a savana parque/savana floresta (cerradão) são importantes habitats dos roedores reservatórios do hantavírus Araraquara (ARAV). Sendo assim, a importância da biodiversidade para o equilíbrio destes ambientes é essencial em razão da manutenção do equilíbrio ecossistêmico, o que evita o "transbordamento" de agentes patógenos para as populações humanas.

Conforme o teste Qui-Quadrado, os resultados do Distrito Federal foram mais expressivos em relação aos impactos antropogênicos e o agravo. A razão disto está no fato de que o espaço do Distrito Federal apresenta um forte dinamismo em sua estrutura, que sugere a criação de um ambiente favorável à enfermidade.

Assim, a criação e preservação de espaços voltados à conservação da biodiversidade do cerrado são mecanismos que colaboram no equilíbrio das populações de roedores silvestres, como, por exemplo, no caso do Necromys lasiurus. Este roedor tem como principais predadores naturais as serpentes e alguns mamíferos carnívoros como o lobo guará e pequenos felinos. A preservação dos predadores de topo, consequentemente irá auxiliar no equilíbrio destas populações de reservatórios de hantavírus em áreas do cerrado. 


\section{O USO E A COBERTURA DA TERRA E A SUA RELAÇÃO COM A HANTAVIROSE NA REGIÃO INTEGRADA DE DESENVOLVIMENTO DO DISTRITO FEDERAL E ENTORNO}

Para as populações rurais, o mais importante para a prevenção da hantavirose é seguir as recomendações preconizadas pelos órgãos de saúde e de extensão rural com a finalidade de que os locais destinados ao armazenamento de insumos e grãos sejam preservados da entrada de roedores sinantrópicos e silvestres. E ainda, faz-se importante a utilização de equipamentos de segurança individual, como, por exemplo, máscaras e luvas que ajudam a realizar atividades laborais de limpeza naqueles ambientes.

De fato, estudos destinados à verificação da densidade de roedores com anticorpos de hantavírus se fazem extremamente necessários no sentido de correlacionar com maior acurácia os locais com a presença de roedores contaminados e, consequentemente, as classes de uso e cobertura da terra que apresentam condições ideais para o desenvolvimento da hantavirose. Seria de grande importância que este tipo de estudo fosse conduzido de forma contínua nas periferias de áreas urbanas tanto do Distrito Federal bem como em sua região de entorno, nas regiões de espaços silvestres.

Finalmente, o presente estudo buscou resgatar a importância do uso espaço no estudo das doenças infectocontagiosas transmitidas por animais vetores e reservatórios de patógenos, uma vez que as doenças ali advindas possuem um elo com atividades humanas e necessitam de abordagem e análise que perpassam a Epidemiologia.

\section{REFERÊNCIAS BIBLIOGRÁFICAS}

ACUNA-SOTO, R., STAHLE, D. W., CLEAVELAND, M. K., THERRELL, M. D. (2002). Megadrought and megadeath in 16th century Mexico. Revista Biomédica, vol. 13, p. 289-292, 2002. <http://www.revistabiomedica.org>. Acesso em: 01 jun. 2012.

AYRES, M., AYRES JÚNIOR, M., AYRES, D. L., SANTOS, A. D. A. (2007). Aplicações estatísticas nas áreas das ciências biomédicas. Belém: Instituto Mamirauá, 2007. 
BREARLEY, G., RHODES, J., BRADLEY, A., BAXTER, G., SEABROOK, L., LUNNEY, D., MCALPINE, C. Wildlife disease prevalence in human-modified landscapes. Biological Reviews, vol. 88, n. 2, p.427-442, 2013. <http:// www. onlinelibrary.wiley.com/>. Acesso em: 15 set. 2014.

BRITO, M.G. Uso da terra e a distribuição espacial de casos humanos da síndrome cardiopulmonar por hantavírus em Minas Gerais, 1996 a 2007. 2012. Tese (doutorado) - Escola de Veterinária, Universidade Federal de Minas Gerais, Belo Horizonte.

EDITORIAL STAFF. Environmental Health Perspective. Vol. 107, n.139,1999. http://citeseerx.ist.psu.edu/viewdoc/download?doi=10.1.1.461.2896\&rep=rep1\&t $\mathrm{ype}=\mathrm{pdf}>$. Acesso em: 10 mar.2014.

estradA-PEÑA, A., OStFeld, R. S., PETERSON, A. T., POULIN, R., de LA FUENTE, J. Effects of environmental change on zoonotic disease risk: an ecological primer. Trends in parasitology, vol. 30, n. 4, p. 205-214, 2014. <http://www.cell.com/trends/parasitology >. Acesso em: 10 jan. 2013.

FOleY, J. A., DEFRIES, R., ASNER, G. P., BARFORD, C., BONAN, G., CARPENTER, S. R., SNYDER, P. K. Global consequences of land use. Science, vol. 309, n.5734, p. 570-574, 2005 . <http:// http://www.sciencemag.org/journals>. Acesso em: 20 jul. 2012.

GLASS, G. E., SHIELDS, T., CAI, B., YATES, T. L., PARMENTER, R. Persistently highest risk areas for hantavirus pulmonary syndrome: potential sites for refugia. Ecological Applications, vol.17, n. 1, p.129-139, 2007. <http:// esajournals.onlinelibrary.wiley.com>. Acesso em: 20 jul. 2012.

GOODIN, D. G., KOCH, D. E., OWEN, R. D., CHU, Y. K., HUTCHINSON, J. M., JONSSON, C. B. (2006). Land cover associated with hantavirus presence in 
Paraguay. Global Ecology and Biogeography, vol.15, n. 5, p. 519-527,2006. <http://onlinelibrary.wiley.com/journal>. Acesso em: 20 fev. 2013.

GOTTDENKER, N. L., STREICKER, D. G., FAUST, C. L., CARROLL, C. R. Anthropogenic land use change and infectious diseases: a review of the evidence. EcoHealth, vol. 11, n.4, p. 619-632., $2014 . \quad<\mathrm{http}: / /$ www.ecohealth.net>. Acesso em: 05 nov. 2014.

INSTITUTO BRASILEIRO DE GEOGRAFIA E ESTATÍSTICA. Manual técnico de uso da terra. Rio de Janeiro: 2013.

LAMBIN, E. F., TRAN, A., VANWAMBEKE, S. O., LINARD, C., SOTI, V. Pathogenic landscapes: interactions between land, people, disease vectors, and their animal hosts. International Journal of Health Geographics, vol. 9, n. 54, p.1-13., 2010. <http:// ij-healthgeographics.biomedcentral.com>. Acesso: 03 mar. 2012.

MINISTÉRIO DA INTEGRAÇÃO NACIONAL. Região Integrada de Desenvolvimento do Distrito Federal e Entorno - RIDE-DF. Disponível em: <http://www.integracao.gov.br/ >. Acesso em: 09 dez. 2014.

MINISTÉRIO DA SAÚDE. Manual de vigilância, prevenção e controle das hantaviroses. Brasília : Ministério da Saúde, 2013.

MINISTÉRIO DA SAÚDE. Surto da síndrome cardiopulmonar por hantavírus no Distrito Federal e Goiás - maio a setembro de 2004. Boletim eletrônico epidemiológico, Brasília, nº 01, 2005.

MINISTÉRIO DO MEIO AMBIENTE. Relatório técnico de monitoramento do desmatamento no cerrado - Bioma Cerrado, 2002 a 2010: Dados Revisados. Centro de Sensoriamento Remoto - CSR/IBAMA. 2011. 
MINISTÉRIO DO MEIO AMBIENTE. Uso e Ocupação do Solo RIDE - 2007. Disponível em: <http://mapas.mma.gov.br// > . Acesso em: 09 dez. 2014.

OLIVEIRA S.V.; ESCOBAR L.E.; PETERSON A.T.; GURGEL-GONÇALVES, R.; Potential Geographic Distribution of Hantavirus Reservoirs in Brazil. PLoS ONE, v. 8, n.12, 2013. <http://www. journals.plos.org/plosntds> Acesso em: 10 mar. 2014.

PATZ, J. A., OLSON, S. H., UEJIO, C. K., GIBBS, H. K. Disease emergence from global climate and land use change. Medical Clinics of North America, vol.92, n. 6, p. 1473-149, 2008. <http:// www.medical.theclinics.com> Acesso em: 20 out. 2011.

SANTOS, J. P., LAVOCAT, M. N., MACHADO, R. R., STEINKE, V. A., STEINKE, E. T. A DINÂMICA DO USO DA TERRA E A HANTAVIROSE. Hygeia, vol. 8, n. 15, 2012. <htpp:// www.hygeia.ig.ufu.br>. Acesso em: 20 set. 2013.

SANTOS, J.P.; STEINKE, E.T.; GARCÍA-ZAPATA, M.T.A. Uso e Ocupação do Solo e a Disseminação da Hantavirose no Distrito Federal, Estudo de Caso: São Sebastião, 2004-2008. Revista da Sociedade Brasileira de Medicina Tropical, v. 44, n. 1, 2011. http://www.sbmt.org.br>. Acesso em: 01 jul. 2011. SANTOS, M. Metamorfoses do Espaço Habitado: Fundamentos Teóricos e Metodológicos da Geografia. São Paulo: EDUSP, 2008.

VAZQUEZ-PROKOPEC, G. M., CEBALLOS, L. A., MARCET, P. L., CECERE, M. C., CARDINAL, M. V., KITRON, U., GÜRTLER, R. E. Seasonal variations in active dispersal of natural populations of Triatoma infestans in rural north-western Argentina. Medical and veterinary entomology, vol. 20, n. 3, p. 273-279, 2006. <http://onlinelibrary.wiley.com/journal/10.1111>. Acesso em: 02 set. 2011 
WU, X., TIAN, H., ZHOU, S., CHEN, L., XU, B. Impact of global change on transmission of human infectious diseases. Science China Earth Sciences, vol.5, n. 2, p. 189-203. 2014. <http://www.springer.com> Acesso em: 10 dez. 2014. 